University of Nebraska - Lincoln

DigitalCommons@University of Nebraska - Lincoln

\title{
Distinctive Hydrocarbons of the Black Dump Fly, Hydrotaea aenescens (Diptera: Muscidae)
}

David A. Carlson

USDA-ARS

Ulrich R. Bernier

University of Florida, ubernier@gainesville.usda.ufl.edu

Jerome A. Hogsette

USDA-ARS

Bruce D. Sutton

Florida Department of Agriculture and Consumer Services

Follow this and additional works at: https://digitalcommons.unl.edu/usdaarsfacpub

Part of the Agricultural Science Commons

Carlson, David A.; Bernier, Ulrich R.; Hogsette, Jerome A.; and Sutton, Bruce D., "Distinctive Hydrocarbons of the Black Dump Fly, Hydrotaea aenescens (Diptera: Muscidae)" (2001). Publications from USDA-ARS / UNL Faculty. 989.

https://digitalcommons.unl.edu/usdaarsfacpub/989

This Article is brought to you for free and open access by the U.S. Department of Agriculture: Agricultural Research Service, Lincoln, Nebraska at DigitalCommons@University of Nebraska - Lincoln. It has been accepted for inclusion in Publications from USDA-ARS / UNL Faculty by an authorized administrator of DigitalCommons@University of Nebraska - Lincoln. 


\title{
Distinctive Hydrocarbons of the Black Dump Fly, Hydrotaea aenescens (Diptera: Muscidae)
}

\author{
David A. Carlson, ${ }^{1 *}$ Ulrich R. Bernier, ${ }^{1}$ Jerome A. Hogsette, ${ }^{1}$ and Bruce D. Sutton ${ }^{2}$ \\ ${ }^{1}$ USDA-ARS, Center for Medical, Agricultural and Veterinary Entomology, Gainesville, Florida \\ ${ }^{2}$ Division of Plant Industry, Florida Department of Agriculture and Consumer Services, \\ Gainesville, Florida
}

\begin{abstract}
Hydrotaea aenescens (Wiedemann), the black dump fly, is a potential biological control agent originally from the western hemisphere, now found in many parts of the Palearctic region except for the United Kingdom, where it cannot be imported for any reason. A complication of classical biological control is the problem of strain identification, as one must be able to somehow mark or follow a particular strain that has been introduced into the field or is contemplated for release. Gas chromatographic analysis of the surface hydrocarbons of pooled and individual dump fly adults resulted in reproducible hydrocarbon patterns that differentiated widely distributed strains of $\boldsymbol{H}$. aenescens and showed similarities between strains that were related. Sexual dimorphism was observed in the surface hydrocarbons. Conspecific similarities included identities of the hydrocarbons found in colony material collected worldwide, with differences being found in the quantities of compounds present. Arch. Insect Biochem. Physiol. 48:167-178, 2001. Published 2001 Wiley-Liss, Inc.
\end{abstract}

\section{Key words: dump fly; identification; biocontrol; house flies; poultry; manure;} cuticular; alkenes

\section{INTRODUCTION}

The black dump fly, Hydrotaea aenescens (Wiedemann), is native to North and South America, and has been used extensively for biological control of house flies, Musca domestica L., in the United States and in various parts of Europe since its introduction in the Palearctic region in the 1970s (Nolan and Kissam, 1985, Ribbeck et al., 1987, Betke et al., 1991, Turner and Carter, 1990, Turner et al., 1992). Its utility for biological control is under active investigation in Florida, where the species is indigenous, but rarely occurs in large numbers (Hogsette and Jacobs, 1999). One advantage of this species as an effective biological control agent is that after establishment in the manure, its larvae destroy house fly larvae effectively, often eliminating this host entirely, probably aided by niche competition in the manure (Hogsette and Jacobs, 1999) . Unlike house flies, dump fly adults tend to stay inside the animal-rearing facilities (Nolan and Kissam, 1987) and do not become pests by gathering in large numbers on the animals, workers (Betke et al., 1991), or on nearby houses and cars (Hogsette and Jacobs, 1999). H. aenescens has been colonized in Florida (Hogsette and Washington, 1995), and was released in large numbers at *Correspondence to: D.A. Carlson, USDA-ARS-CMAVE, P.O.
Box 14565, Gainesville, FL 32607.

E-mail: dcarlson@gainesville.usda.ufl.edu

Received 29 January 2001; Accepted 24 June 2001 
several poultry-rearing facilities for the purpose of controlling populations of house flies. However, recent trials have been confounded by adults absconding from the release site (a pullet farm), and becoming established at a nearby caged-layer farm where no dump flies had been released previously (Hogsette and Jacobs, 1999). Tracing the released dump flies is difficult because no known useful chemical markers, such as rubidium or strontium, can be detected after passing through several generations. Strains of organisms intended for use in biological control efforts are often difficult to identify unless extensive characterization using molecular techniques has been completed. No molecular markers nor visible genetic markers are available for $H$. aenescens to confirm the identities of material in culture, despite the pervasive problem of accidental mixing of cultures among biological control agents.

Dried, intact, or freshly frozen insects may be extracted with organic solvent to obtain waterproofing lipids for chemical analysis. Chemical analysis of a class of these compounds, such as the cuticular hydrocarbons, often yields consistent qualitative and quantitative data showing similarity among the same species and/or sex. Differences in patterns may be seen between closely related or sibling species (Carlson et al., 1993), and sometimes between races (Carlson, 1988a). Detailed composition of the easily obtained cuticular hydrocarbons may serve as an additional tool for taxonomic classification (Carlson, 1988b). These data may be examined using simple peak ratios (Carlson and Service, 1980), or some of the four major types of pattern recognition methodology: mapping and display, clustering, discriminant analysis (Lavine and Carlson, 1987, Lavine et al., 1988), and principal components modeling (Sutton and Carlson, 1993).

Previous studies have clearly established the utility of using hydrocarbon patterns for separations between widely or closely related species of insects with subsequent use of gas chromatography and GC-MS to confirm the identities of compounds detected. Insects studied include Blattella germanica and closely related species (Carlson and Brenner, 1988, Brenner et al., 1993), 26 species and sub-species of tsetse flies (Carlson et al., 1993, Sutton and Carlson, 1997a), and tabanids (Sutton and Carlson, 1997b). Unfortunately, there was only one useful set of museum specimens of $H$. aenescens found at the Florida State Collection of Arthropods, as the only other specimens were in a collection from the 1950s and these flies had been dipped in solvent, probably xylene (B.D. Sutton, personal communication). The hypothesis is that different populations of the species will have sufficiently different hydrocarbon compositions so that they may be classified by multivariate statistical treatment, thus allowing a gas chromatographic protocol to be used to assign individual flies to a particular population. Therefore, we examined cuticular hydrocarbon patterns (CHP) of $H$. aenescens from laboratory colonies and from local study sites where flies had been released, and from additional locations where flies had become established unaided under unknown circumstances. Our point was to determine if flies reared for release could be distinguished from feral populations of unknown origin. For purposes of comparison, specimens were obtained from several worldwide locations to determine the utility of CHP for comparison of populations.

\section{MATERIALS AND METHODS Biological Specimens}

Specimens from our laboratory were removed from colony cages at specific ages, frozen, then separated by sex, and held frozen until utilized in tests. Specimens from a colony in Hungary (collected in Balbona) and a poultry farm in Chile (collected near la Cruz) were held as larvae, and adults were allowed to emerge from pupae held in individual vials; adults died in the vials and were frozen. Adult specimens from a colony in Denmark (collected at Lyngby) were shipped dry. Wild Florida specimens were collected at a large commercial poultry operation, Farm 84 (near Zephyrhills, Pasco County). Gainesville Released specimens were recovered from the field release location one week after release. Pooled and individual specimens were extracted in hexane for 5 min. Dried pinned museum specimens (wild Pensacola) were extracted by removing the labels, then washing the pin with hexane before submerging the pin and specimen in hexane. Specimens have been or will be vouchered at the Florida State Collection of Arthropods, Gainesville. The origin of each group is indicated in Tables 1-5. 
Extracts of cuticular hydrocarbons were further separated on silver nitrate impregnated silica gel to yield fractions containing alkanes and alkenes. The alkene fraction was derivatized by dimethyl disulfide (DMDS) (Francis and Veland, 1981, Carlson et al., 1989).

\section{Chemical Analysis}

The hydrocarbons were isolated from extracted cuticular components using silica gel minicolumns $(50 \times 6 \mathrm{~mm})$ and collecting the first hexane fraction as previously described (Carlson et al., 1984, Carlson and Brenner, 1988). Analysis of cuticular hydrocarbons involved separation and quantitation of eluents by gas chromatography utilizing a fused-silica capillary column (J\&W, $30 \mathrm{~m} \times 0.32 \mathrm{~mm}$ ID, $0.2 \mu \mathrm{m}$ DB-1 stationary phase) fitted to a Hewlett-Packard Model 6890 gas chromatograph, utilizing a cool on-column injector and flame-ionization detector. Hydrogen carrier gas was used at a linear velocity of 40 $\mathrm{cm} / \mathrm{sec}$. Each sample was re-constituted as necessary in $20 \mu \mathrm{l}$ of hexane and 1 to $2 \mu \mathrm{l}$ injected at $60^{\circ} \mathrm{C}$. Each chromatographic run was temperature-programmed as follows: hold for $2 \mathrm{~min}$ $\left(60^{\circ} \mathrm{C}\right)$, ramp $20^{\circ} \mathrm{C} / \mathrm{min}\left(60\right.$ to $\left.230^{\circ} \mathrm{C}\right)$, ramp $2^{\circ} \mathrm{C} /$ min to $320^{\circ} \mathrm{C}$, and hold at $320^{\circ} \mathrm{C}$ to elute all components (10 min). A PC-based data system, Turbochrom 4 (Perkin-Elmer, MA), was used for data recording and quantification, with manual integration of some small peaks, but with some small peaks below $0.02 \%$ not included.

Mass spectra (EI) were obtained using a Hewlett-Packard 5988A MS interfaced to a HP 5890 gas chromatograph fitted with an OCI-3 injector. The chromatographic parameters were as above, except the oven temperature ramp consisted of a 2 -min hold $\left(60^{\circ} \mathrm{C}\right), \operatorname{ramp} 10^{\circ} \mathrm{C} / \mathrm{min}(60$ to $220^{\circ} \mathrm{C}$ ), ramp $3^{\circ} \mathrm{C} / \mathrm{min}$ to $310^{\circ} \mathrm{C}$, and held at $310^{\circ} \mathrm{C}$ to elute all components (approximately 15 $\mathrm{min}$ ). The MS interface was maintained at $310^{\circ} \mathrm{C}$, electron voltage at $70 \mathrm{eV}$, and the system parameters manually optimized in order to enhance the EI spectra in the critical region of 200 to $500 \mathrm{~m} /$ $z$. The mass spectral scan range extended from $\mathrm{m} / \mathrm{z} 35$ to $\mathrm{m} / \mathrm{z} 700$ with a scan rate of 0.75 scan per second. MS scans were manually background subtracted prior to interpretation. Kovat's Retention Indices (KI) were determined by co-injection with normal hydrocarbon standards of 20 to 36 carbons, plus 15,19,23-trimethylheptatriacontane (KI3770). The assignment of KI narrows the range of possible methyl-branch configurations in cases of ambiguous or insufficient EI spectra (Carlson et al., 1998). The identification of cuticular methylalkane components based upon EI mass spectra followed the interpretations established previously (Carlson et al., 1984; Nelson and Sukkestad, 1970; Nelson and Carlson, 1986; Nelson et al., 1976, 1984, 1988).

\section{Discriminant Analysis}

Discriminant analysis utilized linear, quadratic, and kernel density models with brute-force optimization of model parameters using the jackknifed classification error as the decision criteria using the SAS System, version 6.01 (SAS Institute, Cary, NC). The peaks selected for analysis included the predominant isomers of the three dominating homologous series: $\underline{n}$-alkanes, monomethylalkanes, and dimethylalkanes. The baseline for each peak was manually allocated prior to software integration using a predetermined protocol to provide maximum consistency. The individual peak area was expressed as a percentage of the total peak area. Peak subsets were also evaluated as part of the discriminant model optimization (Systat, version 9) (SYSTAT Inc., Evanston, IL).

\section{RESULTS GC Analyses}

Gas chromatographic analysis of all samples showed several structural series of hydrocarbons from 17 to 37 carbons, in which up to 21 of the largest peaks were numbered for all samples and adjusted to $100 \%$. Compounds quantified for these tables included four series: $\underline{n}$-alkanes, methyl branched alkanes, dimethyl branched alkanes, and alkenes (Tables 1-3).

\section{Mass Spectra}

Figure 1 displays the chromatograms for pinned specimens of (a) female and (b) male 1978 Pensacola $H$. aenescens and chromatograms for (c) female and (d) male 1996 Gainesville lab $H$. aenescens. Female flies contained internally branched methyl alkanes with branch points in declining order of intensity at the 13-, 11-, and 
TABLE 1. Percentage Composition ( \pm SE) of Hydrocarbons From H. aenescens: Gainesville Colony*

\begin{tabular}{|c|c|c|c|c|c|c|c|c|c|c|}
\hline$\underline{\mathrm{KI}}$ & $1 d(f) n=5$ & $1 \mathrm{~d}(\mathrm{~m}) \mathrm{n}=5$ & $2 d(f) n=5$ & $2 \mathrm{~d}(\mathrm{~m}) \mathrm{n}=5$ & $3 d(f) n=5$ & $3 \mathrm{~d}(\mathrm{~m}) \mathrm{n}=5$ & $4 \mathrm{~d}(\mathrm{f}) \mathrm{n}=5$ & $4 \mathrm{~d}(\mathrm{~m}) \mathrm{n}=5$ & $8 d(f) n=5$ & $8 \mathrm{~d}(\mathrm{~m}) \mathrm{n}=5$ \\
\hline 2000 & $0.08 \pm 0.01$ & $0.07 \pm 0.01$ & $0.10 \pm 0.01$ & $0.12 \pm 0.01$ & $0.09 \pm 0.01$ & $0.10 \pm 0.01$ & $0.04 \pm 0.01$ & $0.03 \pm 0.01$ & $0.08 \pm 0.01$ & $0.11 \pm 0.01$ \\
\hline 2100 & $0.64 \pm 0.03$ & $0.53 \pm 0.05$ & $0.58 \pm 0.10$ & $0.45 \pm 0.04$ & $0.72 \pm 0.17$ & $0.49 \pm 0.05$ & $1.09 \pm 0.06$ & $0.28 \pm 0.02$ & $2.47 \pm 0.44$ & $0.47 \pm 0.03$ \\
\hline 2200 & $0.15 \pm 0.01$ & $0.15 \pm 0.02$ & $0.22 \pm 0.03$ & $0.23 \pm 0.01$ & $0.21 \pm 0.03$ & $0.26 \pm 0.04$ & $0.22 \pm 0.02$ & $0.09 \pm 0.01$ & $0.40 \pm 0.04$ & $0.37 \pm 0.02$ \\
\hline 2270 & $0.02 \pm 0.01$ & $0.03 \pm 0.01$ & $0.03 \pm 0.01$ & $0.03 \pm 0.01$ & $0.17 \pm 0.13$ & $0.08 \pm 0.02$ & $0.43 \pm 0.10$ & $0.17 \pm 0.05$ & $1.03 \pm 0.29$ & $0.13 \pm 0.02$ \\
\hline 2300 & $0.73 \pm 0.10$ & $0.83 \pm 0.18$ & $5.39 \pm 2.01$ & $2.06 \pm 0.57$ & $6.25 \pm 1.67$ & $4.56 \pm 0.62$ & $18.83 \pm 1.04$ & $3.78 \pm 0.63$ & $24.06 \pm 1.46$ & $5.19 \pm 0.35$ \\
\hline 2400 & $0.28 \pm 0.01$ & $0.35 \pm 0.05$ & $0.46 \pm 0.07$ & $0.40 \pm 0.02$ & $0.29 \pm 0.05$ & $0.39 \pm 0.04$ & $0.42 \pm 0.05$ & $0.33 \pm 0.05$ & $0.44 \pm 0.05$ & $0.52 \pm 0.02$ \\
\hline 2470 & $1.07 \pm 0.48$ & $1.77 \pm 0.68$ & $2.54 \pm 0.41$ & $4.27 \pm 0.95$ & $5.84 \pm 2.06$ & $8.11 \pm 2.19$ & $5.77 \pm 1.02$ & $13.17 \pm 1.76$ & $8.31 \pm 2.25$ & $11.47 \pm 1.02$ \\
\hline 2500 & $5.63 \pm 0.55$ & $7.28 \pm 0.95$ & $11.10 \pm 0.87$ & $11.75 \pm 1.02$ & $7.81 \pm 1.17$ & $10.86 \pm 0.46$ & $13.28 \pm 0.58$ & $14.19 \pm 0.67$ & $8.69 \pm 0.42$ & $12.10 \pm 0.61$ \\
\hline 2600 & $0.90 \pm 0.05$ & $0.90 \pm 0.07$ & $0.76 \pm 0.04$ & $0.76 \pm 0.04$ & $0.45 \pm 0.03$ & $0.51 \pm 0.03$ & $0.70 \pm 0.07$ & $0.41 \pm 0.04$ & $0.54 \pm 0.06$ & $0.44 \pm 0.02$ \\
\hline 2670 & $4.54 \pm 0.50$ & $5.63 \pm 0.74$ & $4.46 \pm 0.79$ & $8.48 \pm 0.73$ & $7.00 \pm 1.57$ & $11.33 \pm 0.97$ & $2.60 \pm 0.57$ & $12.29 \pm 0.90$ & $1.88 \pm 0.45$ & $14.39 \pm 1.11$ \\
\hline 2700 & $16.60 \pm 0.78$ & $14.64 \pm 0.94$ & $12.87 \pm 0.28$ & $13.86 \pm 0.60$ & $9.44 \pm 0.32$ & $9.88 \pm 0.52$ & $15.77 \pm 0.39$ & $10.51 \pm 1.02$ & $15.86 \pm 1.92$ & $8.08 \pm 0.41$ \\
\hline 2735 & $5.57 \pm 0.25$ & $4.94 \pm 0.24$ & $7.75 \pm 0.22$ & $4.19 \pm 0.27$ & $8.58 \pm 1.61$ & $4.71 \pm 1.32$ & $4.31 \pm 0.51$ & $2.29 \pm 0.15$ & $4.24 \pm 0.54$ & $1.48 \pm 0.23$ \\
\hline 2800 & $1.16 \pm 0.04$ & $1.02 \pm 0.13$ & $0.67 \pm 0.03$ & $0.64 \pm 0.04$ & $0.49 \pm 0.02$ & $0.49 \pm 0.04$ & $0.73 \pm 0.09$ & $0.45 \pm 0.05$ & $0.57 \pm 0.05$ & $0.40 \pm 0.02$ \\
\hline 2870 & $30.66 \pm 1.44$ & $32.38 \pm 2.10$ & $22.42 \pm 2.89$ & $31.37 \pm 1.73$ & $24.90 \pm 2.76$ & $29.83 \pm 0.97$ & $5.52 \pm 1.07$ & $26.30 \pm 0.56$ & $2.17 \pm 0.45$ & $29.84 \pm 0.75$ \\
\hline 2900 & $4.94 \pm 0.21$ & $4.10 \pm 0.27$ & $5.07 \pm 0.60$ & $4.71 \pm 0.44$ & $3.74 \pm 0.21$ & $3.88 \pm 0.25$ & $8.39 \pm 0.82$ & $4.64 \pm 0.48$ & $7.73 \pm 0.51$ & $5.19 \pm 0.22$ \\
\hline 2935 & $4.47 \pm 0.17$ & $3.78 \pm 0.12$ & $6.46 \pm 0.24$ & $3.60 \pm 0.14$ & $8.18 \pm 1.57$ & $4.49 \pm 1.15$ & $5.31 \pm 0.61$ & $2.61 \pm 0.15$ & $4.92 \pm 0.79$ & $2.02 \pm 0.21$ \\
\hline 3070 & $15.67 \pm 1.20$ & $15.00 \pm 0.67$ & $11.69 \pm 1.60$ & $9.04 \pm 1.00$ & $8.80 \pm 0.84$ & $6.61 \pm 0.26$ & $4.07 \pm 0.42$ & $6.08 \pm 0.48$ & $1.59 \pm 0.21$ & $5.40 \pm 0.41$ \\
\hline 3100 & $1.51 \pm 0.11$ & $1.56 \pm 0.18$ & $1.72 \pm 0.15$ & $1.35 \pm 0.05$ & $0.99 \pm 0.06$ & $0.94 \pm 0.08$ & $2.16 \pm 0.22$ & $0.94 \pm 0.06$ & $2.21 \pm 0.29$ & $1.07 \pm 0.04$ \\
\hline 3135 & $2.40 \pm 0.13$ & $2.76 \pm 0.79$ & $2.77 \pm 0.38$ & $1.46 \pm 0.07$ & $3.55 \pm 0.77$ & $1.65 \pm 0.42$ & $5.83 \pm 0.59$ & $1.04 \pm 0.08$ & $6.21 \pm 0.40$ & $1.00 \pm 0.07$ \\
\hline 3270 & $1.98 \pm 0.26$ & $1.60 \pm 0.17$ & $1.44 \pm 0.43$ & $0.76 \pm 0.20$ & $0.58 \pm 0.14$ & $0.26 \pm 0.05$ & $0.48 \pm 0.24$ & $0.15 \pm 0.01$ & $0.10 \pm 0.01$ & $0.06 \pm 0.02$ \\
\hline 3335 & $0.55 \pm 0.03$ & $0.46 \pm 0.05$ & $0.59 \pm 0.13$ & $0.30 \pm 0.03$ & $0.80 \pm 0.26$ & $0.29 \pm 0.09$ & $2.75 \pm 0.40$ & $0.17 \pm 0.01$ & $4.52 \pm 0.86$ & $0.21 \pm 0.01$ \\
\hline 3470 & $0.45 \pm 0.15$ & $0.24 \pm 0.04$ & $0.89 \pm 0.31$ & $0.17 \pm 0.02$ & $1.12 \pm 0.37$ & $0.31 \pm 0.17$ & $1.29 \pm 0.16$ & $0.09 \pm 0.01$ & $1.99 \pm 0.22$ & $0.07 \pm 0.01$ \\
\hline
\end{tabular}

*Values represent Mean $\pm \mathrm{SE}$. 
TABLE 2. Percentage Composition $( \pm \mathrm{SE}$ ) of Hydrocarbons from H. aenescens: Gainesville, Hungary, Gainesville/Released, Pensacola, Chile*

\begin{tabular}{|c|c|c|c|c|c|c|c|c|c|c|c|c|}
\hline$\underline{\mathrm{KI}}$ & $\begin{array}{c}\text { Gainesville } \\
4+8 \mathrm{~d}(\mathrm{f}) \mathrm{n}=10\end{array}$ & $\begin{array}{c}\text { Gainesville } \\
4+8 \mathrm{~d}(\mathrm{~m}) \mathrm{n}=10\end{array}$ & $\begin{array}{c}\text { Hungary } \\
4 d(f) n=6\end{array}$ & $\begin{array}{c}\text { Hungary } \\
4 \mathrm{~d}(\mathrm{~m}) \mathrm{n}=6\end{array}$ & $\begin{array}{c}\text { Hungary } \\
8 \mathrm{~d}(\mathrm{f}) \mathrm{n}=6\end{array}$ & $\begin{array}{c}\text { Hungary } \\
8 \mathrm{~d}(\mathrm{~m}) \mathrm{n}=5\end{array}$ & $\begin{array}{l}\text { Gnv/rls } \\
\mathrm{f} \mathrm{n}=15\end{array}$ & $\begin{array}{c}\text { Gnv/rls } \\
\mathrm{m} \mathrm{n}=15\end{array}$ & $\begin{array}{c}\text { Pensacola } \\
\mathrm{f} n=6\end{array}$ & $\begin{array}{c}\text { Pensacola } \\
\mathrm{m} \mathrm{n}=5\end{array}$ & $\begin{array}{c}\text { Chile } \\
\mathrm{fn}=5\end{array}$ & $\begin{array}{c}\text { Chile } \\
\mathrm{m} \mathrm{n}=5\end{array}$ \\
\hline 2000 & $0.06 \pm 0.01$ & $0.07 \pm 0.00$ & $0.09 \pm 0.01$ & $0.27 \pm 0.02$ & $0.10 \pm 0.09$ & $0.31 \pm 0.09$ & $16 \pm 0.08$ & $0.70 \pm 0.08$ & $0.17 \pm 0.05$ & $0.32 \pm 0.05$ & $0.08 \pm 0.00$ & $0.14 \pm 0.05$ \\
\hline 2100 & $1.78 \pm 0.31$ & $0.38 \pm 0.02$ & $0.60 \pm 0.11$ & $0.21 \pm 0.01$ & & & & & & & & \\
\hline 2200 & $0.31 \pm 0.04$ & $0.23 \pm 0.01$ & $0.39 \pm 0.05$ & $0.14 \pm 0.01$ & $0.35 \pm 0$ & & & & & & $0.20 \pm 0.03$ & $0.12 \pm 0.01$ \\
\hline 2270 & $0.73 \pm 0.18$ & $0.15 \pm 0.05$ & $0.23 \pm 0.17$ & $0.16 \pm 0.02$ & $0.48 \pm 0.04$ & $0.19 \pm 0.05$ & $14 \pm 0.04$ & $0.21 \pm 0.07$ & $0.02 \pm 0.01$ & $0.17 \pm 0.06$ & $0.15 \pm 0.07$ & \\
\hline 2300 & $21.45 \pm 1.21$ & $4.48 \pm 0.63$ & $11.05 \pm 2.32$ & $3.40 \pm 0.37$ & $17.55 \pm 0.35$ & $3.72 \pm 0.41$ & $3.17 \pm 0.44$ & & & & 16.79 & 5.78 \\
\hline 2400 & $0.43 \pm 0.03$ & $0.43 \pm 0.05$ & $0.54 \pm 0.07$ & $0.13 \pm 0.01$ & $0.53 \pm 0.21$ & $0.27 \pm 0.03$ & $1.53 \pm 0.56$ & $1.06 \pm 0.20$ & 3.30 & 4.56 & 0.41 & 0.28 \\
\hline 2470 & $7.04 \pm 1.24$ & $12.32 \pm 1.76$ & $6.57 \pm 1.04$ & $11.31 \pm 0.40$ & $8.68 \pm 2.07$ & $14.84 \pm 2.78$ & $2.36 \pm 0.31$ & $2.58 \pm 0.51$ & 0.22 & 0.26 & 4.92 & 13.33 \\
\hline 2500 & $10.99 \pm 0.84$ & $13.14 \pm 0.67$ & $10.20 \pm 0.78$ & $8.86 \pm 0.47$ & $14.46 \pm 1.23$ & $11.54 \pm 0.71$ & $11.93 \pm 0.85$ & $9.37 \pm$ & 21.48 & $19.96 \pm 1.47$ & $15.65 \pm 0.50$ & 16.81 \\
\hline 2600 & $0.62 \pm 0.05$ & $0.43 \pm 0.04$ & $0.91 \pm 0.16$ & $0.26 \pm 0.02$ & $0.64 \pm 0.14$ & $0.32 \pm 0.06$ & $2.07 \pm 0.62$ & $1.96=$ & 2.79 & 2.87 & $0.68=$ & 0.33 \\
\hline 2670 & $2.24 \pm 0.36$ & $13.34 \pm 0.90$ & $3.56 \pm 0.31$ & $14.87 \pm 0.89$ & $3.13 \pm 0.50$ & $14.86=$ & 5.11 & 6.88 & 0.0 & .00 & 2.89 & 12.13 \\
\hline 2700 & $15.82 \pm 0.92$ & $9.30 \pm 1.02$ & $16.91 \pm 1.14$ & $8.07 \pm 0.58$ & $18.61 \pm 0.68$ & $7.91 \pm 0.48$ & $14.74 \pm 0.81$ & $15.36 \pm 0.99$ & $25.74 \pm 2.16$ & 24.91 & $18.79 \pm 1.36$ & 10.63 \\
\hline 2735 & $4.27 \pm 0.35$ & $1.88 \pm 0.15$ & $4.15 \pm 0.42$ & $2.11 \pm 0.13$ & $2.51 \pm 0.09$ & $1.60 \pm 0.12$ & $7.66 \pm 0.53$ & $5.26 \pm 0.32$ & $5.77 \pm 0.58$ & 8.14 & $1.10 \pm 0.14$ & $1.37 \pm 0.05$ \\
\hline 2800 & $0.65 \pm 0.06$ & $0.42 \pm 0.05$ & $1.12 \pm 0.34$ & $0.36 \pm 0.03$ & $0.57 \pm 0.11$ & $0.33 \pm 0.02$ & $3.01 \pm 0.75$ & $3.47 \pm 0.51$ & $2.58 \pm 0.28$ & 0.16 & $0.69 \pm 0.05$ & $0.51 \pm 0.05$ \\
\hline 2870 & $3.85 \pm 0.78$ & $28.07 \pm 0.56$ & $11.63 \pm 2.88$ & $35.76 \pm 0.69$ & $4.81 \pm 0.79$ & $30.22 \pm 2.25$ & $17.12 \pm 1.89$ & $21.27 \pm 1.82$ & $0.16 \pm 0.04$ & $0.41 \pm 0.07$ & $2.51 \pm 0.35$ & $16.56 \pm 1.56$ \\
\hline 2900 & $8.06 \pm 0.47$ & $4.92 \pm 0.48$ & $8.76 \pm 0.77$ & $3.86 \pm 0.31$ & $9.85 \pm 0.31$ & $4.96 \pm 0.28$ & $5.68 \pm 0.33$ & $5.06 \pm 0.23$ & $14.53 \pm 0.48$ & $14.49 \pm 0.73$ & $13.87 \pm 0.78$ & $8.31 \pm 0.68$ \\
\hline 2935 & $5.12 \pm 0.48$ & $2.31 \pm 0.15$ & $4.44 \pm 0.46$ & $1.96 \pm 0.12$ & $2.56 \pm 0.27$ & $1.56 \pm 0.05$ & $7.29 \pm 0.83$ & $8.28 \pm 1.11$ & $6.50 \pm 0.92$ & $6.68 \pm 0.49$ & $1.14 \pm 0.14$ & $1.50 \pm 0.05$ \\
\hline 3070 & $2.83 \pm 0.47$ & $5.74 \pm 0.48$ & $3.82 \pm 0.82$ & $5.67 \pm 0.47$ & $1.41 \pm 0.57$ & $4.70 \pm 0.98$ & $7.61 \pm 0.83$ & $8.43 \pm 0.83$ & $0.24 \pm 0.06$ & $0.44 \pm 0.06$ & $1.41 \pm 0.10$ & $3.86 \pm 0.36$ \\
\hline 3100 & $2.19 \pm 0.17$ & $1.00 \pm 0.06$ & $2.05 \pm 0.23$ & $0.49 \pm 0.02$ & $2.04 \pm 0.03$ & $0.65 \pm 0.08$ & $1.65 \pm 0.14$ & $1.31 \pm 0.09$ & $2.62 \pm 0.16$ & $2.76 \pm 0.21$ & $7.17 \pm 0.85$ & $2.35 \pm 0.19$ \\
\hline 3135 & $6.02 \pm 0.34$ & $1.02 \pm 0.08$ & $5.02 \pm 0.69$ & $0.94 \pm 0.11$ & $3.93 \pm 0.18$ & $0.71 \pm 0.06$ & $4.04 \pm 0.86$ & $2.30 \pm 0.33$ & $4.98 \pm 0.66$ & $4.30 \pm 0.33$ & $2.77 \pm 0.16$ & $1.68 \pm 0.06$ \\
\hline 3270 & $0.29 \pm 0.13$ & $0.11 \pm 0.01$ & $2.54 \pm 0.43$ & $0.88 \pm 0.11$ & $1.51 \pm 0.13$ & $0.71 \pm 0.12$ & $1.15 \pm 0.22$ & $0.95 \pm 0.16$ & $0.06 \pm 0.01$ & $0.25 \pm 0.08$ & $1.55 \pm 0.17$ & $1.44 \pm 0.13$ \\
\hline 3335 & $3.63 \pm 0.54$ & $0.19 \pm 0.01$ & $2.48 \pm 0.49$ & $0.13 \pm 0.02$ & $2.83 \pm 0.06$ & $0.13 \pm 0.01$ & $1.61 \pm 0.39$ & $1.59 \pm 0.14$ & $2.55 \pm 0.32$ & $1.89 \pm 0.18$ & $3.16 \pm 0.21$ & $1.65 \pm 0.10$ \\
\hline 3470 & $1.64 \pm 0.17$ & $0.08 \pm 0.00$ & $2.95 \pm 0.53$ & $0.17 \pm 0.03$ & $2.81 \pm 0.04$ & $0.10 \pm 0.03$ & $0.40 \pm 0.07$ & $0.22 \pm 0.06$ & $3.04 \pm 0.46$ & $0.76 \pm 0.42$ & $2.33 \pm 0.30$ & $0.66 \pm 0.15$ \\
\hline
\end{tabular}

*Values represent Mean $\pm \mathrm{SE}$. 
TABLE 3. Percentage Composition ( \pm SE) of Hydrocarbons From $H$. aenescens: Zephyrhills, Denmark*

\begin{tabular}{|c|c|c|c|c|c|c|c|c|}
\hline$\underline{\mathrm{KI}}$ & $\begin{array}{c}\text { Zephyr } \\
4 \mathrm{~d}(\mathrm{f}) \mathrm{n}=2\end{array}$ & $\begin{array}{c}\text { Zephyr } \\
4 \mathrm{~d}(\mathrm{~m}) \mathrm{n}=5\end{array}$ & $\begin{array}{c}\text { Zephyr } \\
8 d(f) n=2\end{array}$ & $\begin{array}{c}\text { Zephyr } \\
8 \mathrm{~d}(\mathrm{~m}) \mathrm{n}=5\end{array}$ & $\begin{array}{c}\text { Denmark } \\
5 d(f) n=6\end{array}$ & $\begin{array}{c}\text { Denmark } \\
5 \mathrm{~d}(\mathrm{~m}) \mathrm{n}=6\end{array}$ & $\begin{array}{c}\text { Denmark } \\
8 d(f) n=6\end{array}$ & $\begin{array}{c}\text { Denmark } \\
8 \mathrm{~d}(\mathrm{~m}) \mathrm{n}=6\end{array}$ \\
\hline 2000 & $0.88 \pm 0.04$ & $0.37 \pm 0.10$ & $0.39 \pm 0.08$ & $0.56 \pm 0.09$ & $0.01 \pm 0.01$ & $0.01 \pm 0.01$ & $0.01 \pm 0.01$ & $0.01 \pm 0.01$ \\
\hline 2100 & $2.13 \pm 0.10$ & $0.95 \pm 0.18$ & $1.37 \pm 0.30$ & $1.25 \pm 0.21$ & $0.57 \pm 0.06$ & $0.30 \pm 0.01$ & $0.62 \pm 0.05$ & $0.32 \pm 0.02$ \\
\hline 2200 & $1.34 \pm 0.04$ & $0.93 \pm 0.22$ & $1.32 \pm 0.34$ & $1.32 \pm 0.29$ & $0.28 \pm 0.02$ & $0.21 \pm 0.02$ & $0.33 \pm 0.03$ & $0.21 \pm 0.02$ \\
\hline 2270 & $0.49 \pm 0.21$ & $0.27 \pm 0.08$ & $0.30 \pm 0.02$ & $0.26 \pm 0.04$ & $0.37 \pm 0.04$ & $0.26 \pm 0.02$ & $0.79 \pm 0.15$ & $0.25 \pm 0.02$ \\
\hline 2300 & $14.93 \pm 1.39$ & $3.99 \pm 0.20$ & $16.61 \pm 5.01$ & $4.77 \pm 0.35$ & $7.47 \pm 0.61$ & $2.65 \pm 0.23$ & $14.61 \pm 1.07$ & $4.64 \pm 0.51$ \\
\hline 2400 & $1.13 \pm 0.13$ & $0.97 \pm 0.13$ & $1.29 \pm 0.20$ & $1.16 \pm 0.21$ & $0.51 \pm 0.05$ & $0.48 \pm 0.06$ & $0.62 \pm 0.06$ & $0.39 \pm 0.03$ \\
\hline 2470 & $2.10 \pm 0.27$ & $12.90 \pm 1.75$ & $1.12 \pm 0.02$ & $11.60 \pm 2.07$ & $7.35 \pm 0.77$ & $10.60 \pm 1.05$ & 1.68 & 12.5 \\
\hline 2500 & $11.03 \pm 5.35$ & $13.76 \pm 0.84$ & $7.66 \pm 3.44$ & $12.99 \pm 1.23$ & $11.42 \pm 0.44$ & 10.0 & $12.71 \pm 0.81$ & 0.60 \\
\hline 2600 & $0.92 \pm 0.14$ & $0.84 \pm 0.10$ & $1.23 \pm 0.17$ & $0.77 \pm 0.14$ & $0.82 \pm 0.07$ & 0.11 & $0.87 \pm 0.08$ & 0.07 \\
\hline 2670 & $4.63 \pm 0.96$ & $10.76 \pm 0.95$ & $1.67 \pm 0.80$ & $11.90 \pm 0.50$ & $7.36 \pm 0.49$ & $14.57 \pm 1.04$ & $5.75 \pm 0.89$ & 14.79 \\
\hline 2700 & $13.70 \pm 0.52$ & $8.28 \pm 0.69$ & $20.60 \pm 3.60$ & $7.02 \pm 0.68$ & $15.01 \pm 0.82$ & $12.10 \pm 0.88$ & $17.24 \pm 1.06$ & $8.17 \pm 0.41$ \\
\hline 2735 & $5.42 \pm 0.78$ & $2.28 \pm 0.07$ & $6.13 \pm 2.80$ & $1.36 \pm 0.09$ & $5.19 \pm 0.36$ & $2.57 \pm 0.14$ & $2.06 \pm 0.12$ & $1.49 \pm 0.14$ \\
\hline 2800 & $0.39 \pm 0.08$ & $0.29 \pm 0.04$ & $0.50 \pm 0.41$ & $0.40 \pm 0.11$ & $1.03 \pm 0.24$ & $0.75 \pm 0.09$ & $0.56 \pm 0.09$ & $0.78 \pm 0.23$ \\
\hline 2870 & $12.72 \pm 2.46$ & $27.45 \pm 1.10$ & $4.58 \pm 0.81$ & $28.26 \pm 0.79$ & $15.90 \pm 1.27$ & $28.69 \pm 0.62$ & $9.27 \pm 1.14$ & $31.22 \pm 1.24$ \\
\hline 2900 & $5.80 \pm 0.64$ & $3.29 \pm 0.14$ & $13.63 \pm 0.81$ & $4.33 \pm 0.31$ & $5.68 \pm 0.63$ & $4.37 \pm 0.57$ & $8.24 \pm 0.61$ & $3.71 \pm 0.22$ \\
\hline 2935 & $6.00 \pm 0.55$ & $2.73 \pm 0.34$ & $6.32 \pm 1.81$ & $2.06 \pm 0.27$ & $4.85 \pm 0.22$ & & & $1.51 \pm 0.13$ \\
\hline 3070 & & $6.72 \pm 1.00$ & & 6.5 & & & & \\
\hline 3100 & $1.11 \pm 0.18$ & $0.50 \pm 0.04$ & $2.36 \pm 0.34$ & $0.82 \pm 0.03$ & $1.32 \pm 0.30$ & 0.58 & 1.64 & $0.55 \pm 0.03$ \\
\hline 3135 & $4.91 \pm 0.24$ & $1.22 \pm 0.21$ & $5.34 \pm 0.07$ & $1.22 \pm 0.18$ & $3.81 \pm 0.30$ & $1.07 \pm 0.11$ & $3.30 \pm 0.41$ & $0.94 \pm 0.05$ \\
\hline 3270 & $2.11 \pm 0.22$ & $0.97 \pm 0.17$ & $1.75 \pm 0.13$ & $0.99 \pm 0.13$ & $2.19 \pm 0.19$ & $1.60 \pm 0.16$ & $1.82 \pm 0.16$ & $1.53 \pm 0.17$ \\
\hline 3335 & $1.83 \pm 0.11$ & $0.32 \pm 0.05$ & $2.45 \pm 0.06$ & $0.37 \pm 0.06$ & $1.64 \pm 0.22$ & $0.35 \pm 0.04$ & $2.37 \pm 0.42$ & $0.40 \pm 0.01$ \\
\hline 3470 & $1.63 \pm 0.60$ & $0.19 \pm 0.03$ & $1.38 \pm 0.44$ & $0.09 \pm 0.04$ & $3.05 \pm 0.21$ & $0.61 \pm 0.04$ & $2.70 \pm 0.15$ & $0.59 \pm 0.03$ \\
\hline
\end{tabular}

*Values represent Mean $\pm \mathrm{SE}$.

15- positions for the larger odd-backbone alkanes at C27, C29, C31, C33, C35, and C37. There were much smaller amounts (less than $0.1 \%$ ) of 9-, 7-, 5-, 3-, and 2- methyl alkanes and even-backboned alkanes with methyl branching corresponding to those of the major components that were omitted from the results of GC analyses found in Tables $1-3$. These same internally branched components were present but in much reduced quantities in corresponding males as can be seen in the Gainesville and Hungary flies for KI 3335 and KI 3535, identified as monomethyl branched alkanes (Table 4). The unsaturated hydrocarbons from these flies were of the $(Z)$ - configuration as shown by argentation TLC and comparison with (Z)-9-tricosene standard. Nearly all unsaturated hydrocarbons were shown to be 9 -alkenes by DMDS derivatization and GC-MS, with all males showing a predominant (Z)-9-C29:1 peak (eluting at KI 2870) as the largest hydrocarbon, with notation as shown in Table 4. Specifically, Florida 1978 males (Pensacola) included the following major alkenes: (Z)-9-C25:1 (KI 2470), (Z)-9-C29:1 (KI 2870 ), with lesser amounts of $(Z)-9-\mathrm{C} 27: 1$ (KI 2670), (Z)-9- and 8-C26:1 (KI 2570), (Z)-9-C31:1 (KI 3070), (Z)-9-C33:1 (KI 3270) and trace amounts of $(9, \mathrm{x}-)$ dienes that appeared to be $(Z, Z)-9,18-\mathrm{C} 25: 2,9,18-\mathrm{C} 27: 2,9,18-\mathrm{C} 29: 2$, and 9,18-C31:2. These dienes are expected to be $(Z, Z)$-dienes (Bartelt et al., 1982) but no further characterization was attempted. The unsaturated hydrocarbons from corresponding Pensacola females included major amounts of (Z)-9-C29:1 (KI 2870) and (Z)-9-C31:1 (KI 3070) and smaller amounts of the same alkenes. Wild Florida males (1996, Farm 84) showed a major alkene peak at (Z)-9-C29:1 (KI 2870) and large amounts of (Z)-9-C25:1 (KI 2470), (Z)-9-C27:1 (KI 2670), and (Z)-9-C31:1 (KI 3070). Corresponding wild Florida females (1996, Farm 84) showed nearly equal quantities of the same alkenes but with the addition of $(Z)$-alkenes at $(Z)$ 9-C23:1 (KI 2270), (Z)-9-C24:1 (KI 2370), and (Z)-9-C35:1 (KI 3470). Wild Florida females (1996) were similar and showed nearly equal quantities of these alkenes again with the addition of small amounts of $(Z)$ - alkenes at $(Z)$ 9-C23:1 (KI 2270), (Z)-9-C24:1 (KI 2370), and (Z)-9-C35:1 (KI 3470). A transition from 3methylene interrupted dimethylalkanes (e.g. $\left.11,15-\mathrm{Me}_{2} \mathrm{C}_{25}\right)$ to 11-methylene interrupted dimethylalkanes (e.g., $11,21-\mathrm{Me}_{2} \mathrm{C}_{33}$ ) occurs at C31, where both 3- and 11- methylene inter- 

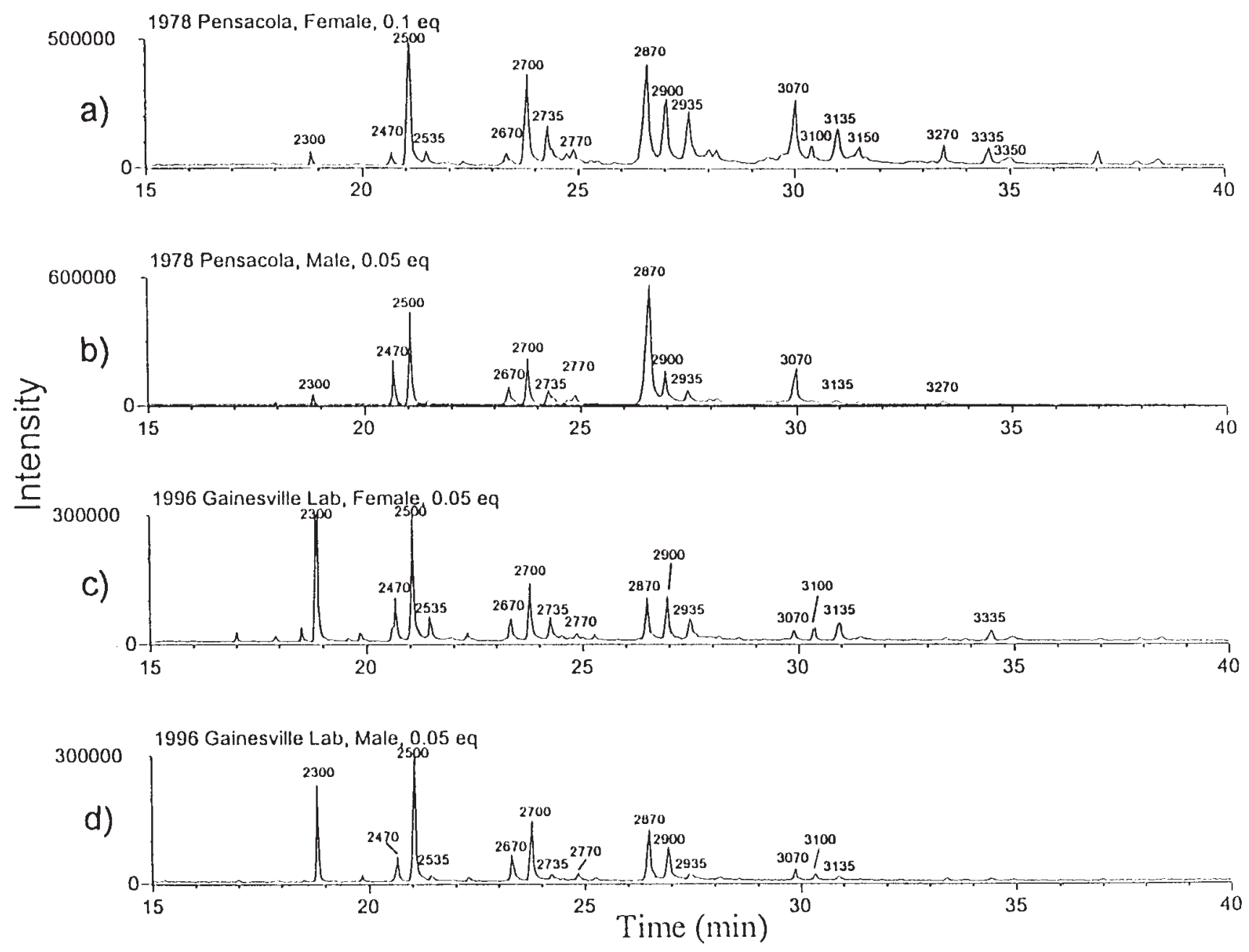

Fig. 1. Total ion current chromatograms from electron ionization GC-MS of (a) pinned 1978 female $H$. aenescens from Pensacola, (b) pinned 1978 male $H$. aenescens from Pen-

rupted dimethylalkanes are present. Dimethylalkanes of both 3- and 11-methylene interruptions are present at C31; normally, only one interruption pattern is seen for a particular chain length, such as the transition from 3-methylene interruptions at $\mathrm{C} 34$, to 11-methylene interruptions at C35 in Musca domestica (Nelson et al. 1981). The identities of 55 peaks detected in GC-MS analysis of these flies are presented in Table 4.

\section{Aged Flies Study}

Hydrocarbons from Gainesville males and females were analyzed as individual samples (Table 1). Changes seen with age included the following trends comparing percent composition after the first day: in males several of the larger alkane components stayed about the same (KI sacola, (c) 1996 female $H$. aenescens from the Gainesville lab colony, and (d) 1996 male $H$. aenescens from the Gainesville lab colony.

$2500,2700,2900)$ and others about doubled with the alkenes gaining most (KI 2370, 2470, 2670). One alkane declined by about half with the higher alkenes declining even more (KI 2700, 3070, 3270). In females, several of the larger alkane peaks stayed nearly constant (KI 2500, 2700), some gained dramatically (KI $2300[5 \times])$ as did two minor alkenes (KI 2270 [5×], 2470 [3×]). Some declined by about half (KI 2670) with the higher alkenes declining even more (KI 3070 [15×], 3070 $[7 \times]$, and $3270[15 \times])$. The increasing and decreasing trends generally appeared to continue from the quantities found on day one. As it is difficult to describe the composition of an "average" fly other than by averaging, all of these data were employed for the discriminant analysis presented below. 
174 Carlson et al.

TABLE 4. Identities of Hydrotaea aenescens Cuticular Hydrocarbons Identified by GC-MS*

\begin{tabular}{|c|c|c|c|}
\hline$\underline{\mathrm{KI}}$ & Hydrocarbons & H. aenescens $\mathrm{f}$ & H. aenescens $\mathrm{n}$ \\
\hline 2000 & $\mathrm{n}-\mathrm{C}_{20}$ & $\mathrm{t}$ & $\mathrm{t}$ \\
\hline 2100 & $\mathrm{n}-\mathrm{C}_{21}$ & + & $\mathrm{t}$ \\
\hline 2200 & $\mathrm{n}-\mathrm{C}_{22}$ & + & + \\
\hline 2270 & (Z)-9- $\mathrm{C}_{23: 1}$ & $\mathrm{t}$ ? & $\mathrm{t}$ ? \\
\hline 2300 & $\mathrm{n}-\mathrm{C}_{23}$ & + & + \\
\hline 2335 & $11-\mathrm{MeC}_{23}$ & $\mathrm{t}$ ? & - \\
\hline 2400 & $\mathrm{n}-\mathrm{C}_{24}$ & + & + \\
\hline 2470 & (Z) $-9-\mathrm{C}_{25: 1}$ & $\mathrm{t}$ & + \\
\hline 2500 & $\mathrm{n}-\mathrm{C}_{25}$ & + & + \\
\hline 2535 & $<\mathrm{S}>13-\mathrm{MeC}_{25}, 11-\mathrm{MeC}_{25}, 9-\mathrm{MeC}_{25}$ & +++ & +++ \\
\hline 2555 & $<\mathrm{S}>11,15-\mathrm{Me}_{2} \mathrm{C}_{25}, 9,13-\mathrm{Me}_{2} \mathrm{C}_{25}$ & $\mathrm{t} ? \mathrm{t} ?$ & -- \\
\hline 2560 & $2-\mathrm{MeC}_{25}$ & + & + \\
\hline 2570 & $7,11-\mathrm{Me}_{2} \mathrm{C}_{25}$ & $\mathrm{t}$ & - \\
\hline 2575 & $3-\mathrm{MeC}_{25}$ & + & + \\
\hline 2600 & $\mathrm{n}-\mathrm{C}_{26}$ & + & + \\
\hline 2635 & $13-\mathrm{Me}_{2} \mathrm{C}_{26}$ & + & - \\
\hline 2670 & (Z)-9-C $27: 1$ & + & + \\
\hline 2700 & $\mathrm{n}-\mathrm{C}_{27}$ & + & + \\
\hline 2735 & $13-\mathrm{MeC}_{27}, 11-, \mathrm{MeC}_{27}, 9-\mathrm{MeC}_{27}$ & +++ & +++ \\
\hline 2740 & $7-\mathrm{MeC}_{27}$ & + & + \\
\hline 2750 & $5-\mathrm{MeC}_{27}$ & + & $\mathrm{t}$ \\
\hline 2755 & $11,15-\mathrm{Me}_{2} \mathrm{C}_{27}, 7,11-\mathrm{Me}_{2} \mathrm{C}_{27}$ & $\mathrm{t} ? \mathrm{t}$ ? & + \\
\hline 2760 & $2-\mathrm{MeC}_{27}$ & + & + \\
\hline 2770 & (Z)-9- $\mathrm{C}_{28: 1}$ & - & + \\
\hline 2775 & $3-\mathrm{MeC}_{27}$ & + & + \\
\hline 2800 & $\mathrm{n}-\mathrm{C}_{28}$ & + & $\mathrm{t}$ \\
\hline 2835 & $14-\mathrm{MeC}_{28}$ & + & - \\
\hline 2870 & (Z)-9- $\mathrm{C}_{29: 1}$ & + & + \\
\hline 2900 & $\mathrm{n}-\mathrm{C}_{29}$ & + & + \\
\hline 2935 & $13-\mathrm{MeC}_{29},<\mathrm{S}>\mathrm{MeC}_{29}, 11-\mathrm{MeC}_{29}, 9-\mathrm{MeC}_{29}$ & +++ & +++ \\
\hline 2940 & $7-\mathrm{MeC}_{29}$ & + & - \\
\hline 2955 & $11,5-\mathrm{Me}_{2} \mathrm{C}_{29},<\mathrm{S}>13,17-\mathrm{Me}_{2} \mathrm{C}_{29}, 9,13-\mathrm{Me}_{2} \mathrm{C}_{29}$ & +++ & +++ \\
\hline 2960 & $2-\mathrm{MeC}_{29}$ & + & + \\
\hline 2975 & $3-\mathrm{MeC}_{29}$ & + & + \\
\hline 3000 & $\mathrm{n}-\mathrm{C}_{30}$ & + & - \\
\hline 3035 & $12-\mathrm{MeC}_{30}$ & + & - \\
\hline 3055 & $(\mathrm{Z}, \mathrm{Z})-9,18-\mathrm{C}_{31: 2}$ & + & + \\
\hline 3070 & (Z)-9- $\mathrm{C}_{31: 1}$ & + & + \\
\hline 3100 & $\mathrm{n}-\mathrm{C}_{31}$ & $\mathrm{t}$ & - \\
\hline 3135 & $11-\mathrm{MeC}_{31}, 13-\mathrm{MeC}_{31}, 15-\mathrm{MeC}_{31}, 9-\mathrm{MeC}_{31}$ & ++++ & +++- \\
\hline 3140 & $7-\mathrm{MeC}_{31}$ & $\mathrm{t}$ & - \\
\hline \multirow[t]{2}{*}{3155} & $13,17-\mathrm{Me}_{2} \mathrm{C}_{31}, 11,15-\mathrm{Me}_{2} \mathrm{C}_{31}, 9,13-\mathrm{Me}_{2} \mathrm{C}_{31}$ & +++ & --- \\
\hline & $<\mathrm{S}>11,21-\mathrm{Me}_{2} \mathrm{C}_{31}, 9,19-\mathrm{Me}_{2} \mathrm{C}_{31}$ & $+\mathrm{t}$ & $-\mathrm{t}$ \\
\hline 3160 & $2-\mathrm{MeC}_{31}$ & + & + \\
\hline 3175 & $3-\mathrm{MeC}_{31}$ & + & $\mathrm{t}$ \\
\hline 3255 & $(\mathrm{Z}, \mathrm{Z})-9,18-\mathrm{C}_{33: 2}$ & + & + \\
\hline 3270 & (Z)-9- $\mathrm{C}_{33: 1}$ & + & + \\
\hline 3335 & $11-\mathrm{MeC}_{33}, 13-\mathrm{MeC}_{33}, 15-\mathrm{MeC}_{33}, 9-\mathrm{MeC}_{33}$ & ++++ & +++- \\
\hline \multirow[t]{2}{*}{3355} & $<\mathrm{S}>15,19-\mathrm{Me}_{2} \mathrm{C}_{33}, 13,17-\mathrm{Me}_{2} \mathrm{C}_{33}, 11,15-$ & $t++$ & +++ \\
\hline & $\mathrm{Me}_{2} \mathrm{C}_{33}, 11,21-\mathrm{Me}_{2} \mathrm{C}_{33},<\mathrm{S}>15,19-\mathrm{Me}_{2} \mathrm{C}_{33}, 9,19-\mathrm{Me}_{2} \mathrm{C}_{33}$ & $++\mathrm{t} ?$ & $\mathrm{t} ?--$ \\
\hline 3470 & $(\mathrm{Z})-9-\mathrm{C}_{35: 1}$ & + & - \\
\hline 3535 & $13-\mathrm{MeC}_{35}, 15-\mathrm{MeC}_{35}, 11-\mathrm{MeC}_{35}$ & +++ & --- \\
\hline 3555 & $<\mathrm{S}>13,23-\mathrm{Me}_{2} \mathrm{C}_{35}, 11,21-\mathrm{Me}_{2} \mathrm{C}_{35}$ & ++ & -- \\
\hline 3670 & (Z)-9- $\mathrm{C}_{37: 1}$ & + & - \\
\hline 3755 & $13,23-, 11,21-\mathrm{Me}_{2} \mathrm{C}_{37}$ & ++ & -- \\
\hline 3955 & $13,23-\mathrm{Me}_{2} \mathrm{C}_{39}$ & + & - \\
\hline 4155 & $13,23-\mathrm{Me}_{2} \mathrm{C}_{41}$ & $\mathrm{t}$ & - \\
\hline
\end{tabular}

* $<\mathrm{S}>$, symmetrical; $\mathrm{t}$, trace amount of compound; ?, uncertainty as to the identity; KI, adjusted to produce uniform indices for $\mathrm{C}_{35}+$; female; $\mathrm{m}$, male. 


\section{Comparison of Patterns in Hydrocarbons from Females}

In order to display consistency of hydrocarbon components, the CHP of 5 groups of females are compared (Fig. 2). Consistent results were seen in patterns from Gainesville (4 plus $8 \mathrm{~d}$ ), Hungary (4 plus 8d), and Chile females in which 10 peaks were consistently present in about the same proportions in females except for KI 3270 in Gainesville females. These ten peaks were chosen because they were much larger in females than in males for all 10 peaks except KI 2270 in Hungary (4d), and KI 2270 and KI 3270 in Chile females, in which these peaks were small and equal in both sexes (Table 2). The comparison of the same 10 peaks of this group of three suggested less similarity with both Gainesville Released females (smaller alkane peak at KI 2300 and smaller alkene peaks at KI 2270 and KI 3470) and Pensacola females (smaller alkene peaks at KI 2270 and KI 3270). The CHP of these 10 peaks showed little sexual dimorphism compared to males (Table 2). Results from other CHP sets of $4 \mathrm{~d}$ Zephyrhills females showed the same trends, as 9 of 10 peaks were larger in females than in corresponding males except for the KI 2900 peak, and the 8-d females had 8 of 10 larger with KI 2100 and KI 2270 nearly the same intensity (Table 3). The CHP from both 4- and 8-d Denmark females showed 10 of 10 peaks larger than in males. In Zephyrhills and Denmark males, three alkene peaks (KI 2470, 2670, and 2870) were consistently larger than in corresponding females (Table 3).

\section{Discriminant Analysis}

The results of the three-way linear discriminant model for all ages of the Gainesville laboratory (Table 1) and all ages of the Hungary flies and Gainesville Released flies using 22 peaks (Table 2) showed that the three samples had jackknifed classification scores of $100 \%$ (Gainesville Colony), 96\% (Hungary), and 93\% (Gainesville Released) (Table 5). For both the Hungary and Released samples, misclassified individuals ( $\mathrm{n}=$ 1 and 2, respectively) were classified as Gainesville. No Gainesville or Released individuals were classified as Hungary. This analysis used data from all ages of flies and therefore ignored sexual dimorphism in hydrocarbon patterns and changes with age. The canonical scores plot (Fig. 3) shows the $95 \%$ ellipsoids to be disjoint and well sepa-

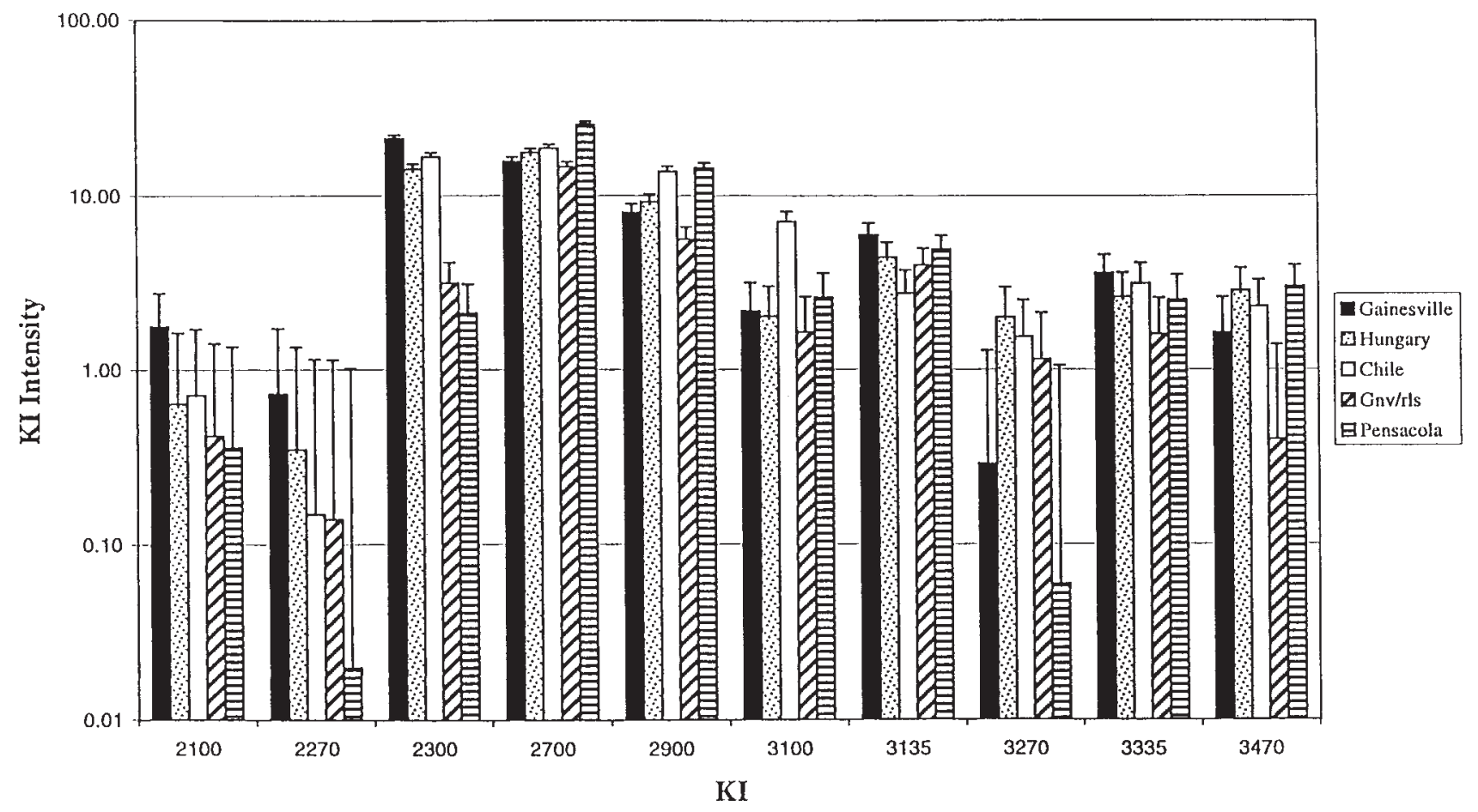

Fig. 2. Comparison of hydrocarbon \% composition from H. aenescens females: Gainesville, Hungary, Chile, Gainesville Released, Pensacola 
TABLE 5. Jackknifed Classification Scores for the Linear Discriminant Model: Gainesville Colony, Gainesville Released, and Hungary, Using Hydrocarbon Composition

\begin{tabular}{lcccc}
\hline & $\begin{array}{c}\text { Gainesville } \\
\text { Colony }\end{array}$ & $\begin{array}{c}\text { Gainesville } \\
\text { Hungary }\end{array}$ & Released & $\begin{array}{c}\% \\
\text { Correct }\end{array}$ \\
\hline Gainesville & 50 & 0 & 0 & 100 \\
$\quad$ Colony & & 22 & 0 & 96 \\
Hungary & 1 & 0 & 27 & 93 \\
Gainesville & 2 & 22 & 27 & 97 \\
$\quad \begin{array}{l}\text { Released } \\
\text { Total }\end{array}$ & 53 & & & \\
\hline
\end{tabular}

rated, and indicates that the Gainesville and Released samples had somewhat more similar hydrocarbon patterns than the Released and Hungary flies. A two-way linear discriminant model based on the Gainesville and Hungary samples classified $100 \%$ of the released flies as Gainesville (not shown) that is consistent with the Gainesville Released flies being somewhat more consistent with Gainesville Colony than with Hungary flies.

\section{DISCUSSION}

Multivariate methods were used as a standalone comparison of the data here, since these meth- ods are regularly used for pattern recognition. The peaks used for MDS were identified by KI numbers as a convenient shorthand, since no behavioral element is assigned to them, and their identities are provided in Tables 1-5 for comparison.

We conclude that CHPs were consistent among the colony flies of known ages, with dimorphism becoming more pronounced with age, as shown in results from older females and males. CHPs showed sexual dimorphism at 4 to 8 days that was shown to be remarkably consistent among Gainesville, Zephyrhills, Hungary, Chile, and Denmark females. The Pensacola and Gainesville Released flies were of unknown ages, and maybe young, when the CHP dimorphism is less pronounced. Compared with corresponding females, 4 alkenes were observed as large or major peaks that were consistently larger in males, including KI 2470, 2670, 2870, and usually 3070, for older Gainesville (8d), Zephyrhills, all Hungary and Chile males. In the Gainesville Released flies, these peaks were present, but there were essentially no differences in the composition of these male-dominant alkenes between the sexes, suggesting that the flies were recaptured when very young.

The Gainesville Released flies had been in

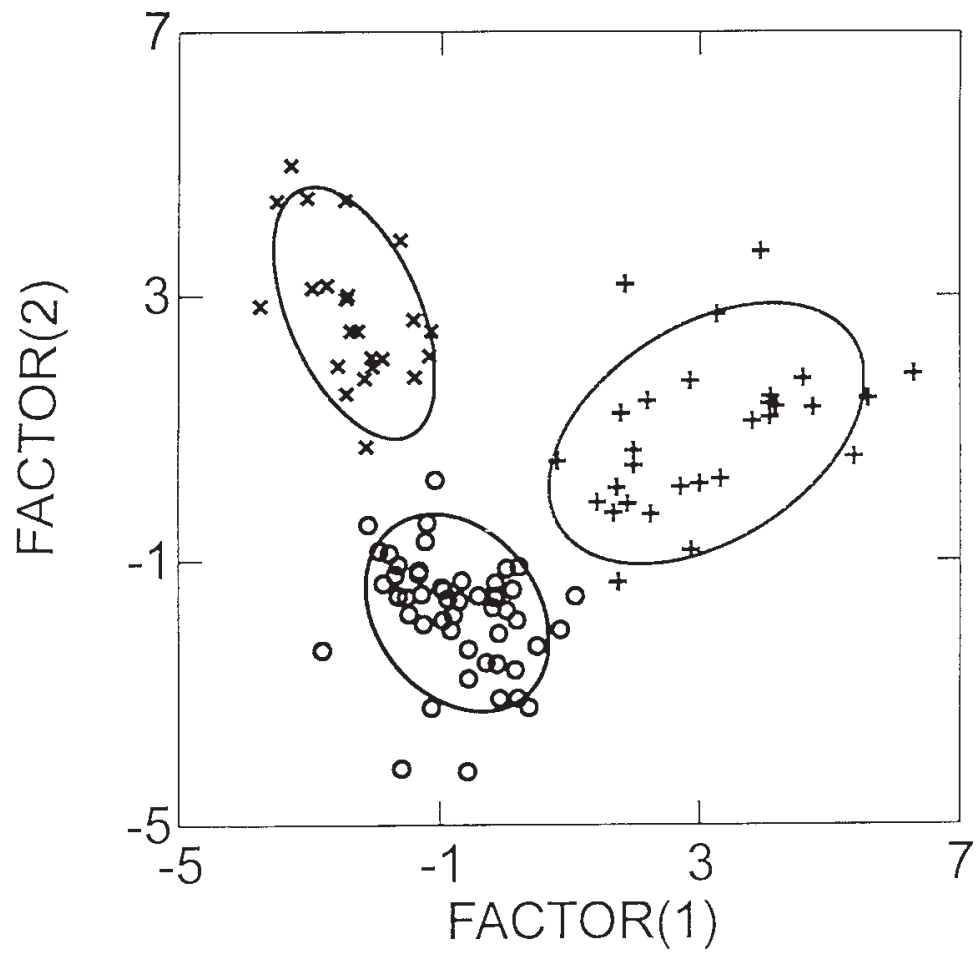

Fig. 3. Canonical scores plot using hydrocarbon \% composition from Gainesville, Gainesville Released and Hungary flies. 
culture for at least 5 years prior to release, but were expected to become established at the apparently satisfactory release site. However, because they did not, for unknown reasons, become established, there were no adults or larvae produced from this site for comparative chemical analysis. We used the colony specimens retained at that time to represent the specimens released. Because $H$. aenescens was released at the pullet farm in such large numbers ( $>2$ million) over a 14-month period, it seems reasonable to assume that their offspring were the same flies that became established nearby (within 300 meters) at a similar type of poultry production facility. This was an unexpected circumstance, because no dump flies had been observed or collected at the second location before the releases began (Hogsette and Jacobs, 1999).

Sexual dimorphism was evident in CHP of $H$. aenescens, with the male CHP being simpler in each instance. It was encouraging to observe that siblings of a particular strain retained very nearly the same CHP regardless of origin and the patterns were reproduceable. We have investigated and described the effects of age up to 8 days on fresh specimens; however, the possible effect of age on museum specimens is not known. The effects of rearing conditions upon the composition of hydrocarbons are not known, although the hydrocarbons described here are the result of biosynthesis by the insects, since these materials are unlikely to be present in their rearing media.

Storage of samples as crude extracts, or as chromatographically purified alkanes had no effect on the results. After solvent-free dried samples were stored for one year at room temperature, some samples were re-analyzed and virtually the same chromatograms were obtained. Thus, samples such as those described here are not obviously affected by age or reasonable condition of storage. It is preferable to dry and store specimens in covered glass containers, or frozen in glass vials although Eppendorf tubes did not give false artifacts. This technique can give rapid results with dried material, perhaps allowing advantages over a species-diagnostic genetic marker RAPD-PCR technique, which in any case is not available for this insect.

The Multi-Dimensional Scaling and discriminant analysis results were not consistent with the assumption that the flies captured at the new site were of the same strain as the released flies. It is possible, if unlikely, that the flies sampled from the new site, a poultry-rearing facility, originated from a source other than those released, i.e., wild progenitors. Alternatively, there may have been a shift in CHP associated with the release of the colony flies into a radically different environment compounded, perhaps, by a founder effect. The captured flies did not exhibit the same degree of sexual dimorphism as seen in the laboratoryreared flies. This could be the result of sampling a single age class of recently eclosed adults; however, the lack of any overlap between the clusters of laboratory and captured flies suggests that age bias is not in itself sufficient to explain the results. The phenomenon of development of sexual dimorphisn in CHP has been observed previously in house flies, in which $(Z)-9-C 23: 1$ (the sex pheromone ( $Z$ )-9-tricosene) is small or undetectable in females of some wild strains, but is prominent or becomes the major peak in some wild strains and in virtually all colonized house flies (Adler et al., 1984, D.A. Carlson, unpublished data). Curiously, we found that (Z)-9-C25:1 is a major component in dump flies, apparently due to different chain-length specificity of elongation enzymes compared to other muscid flies such as house flies, which have very little of this alkene.

\section{ACKNOWLEDGMENTS}

We thank Dr. Jorgen B. Jespersen, Danish Pest Infestation Laboratory, Lyngby, Denmark, Dr. Robert Farkas, Szent Istvan University, Faculty of Veterinary Science, Budapest, Hungary, and Dr. Renato Ripa, Institute of Agricultural Research, La Cruz, Chile, for sending dump fly samples for use in this project. We thank M. Hosack for technical assistance and M. Falkner for data and manuscript preparation.

\section{LITERATURE CITED}

Adler V, Uebel EC, Schwarz M. 1984. Production of (Z)-9tricosene by colonized and feral female house flies. Southwest Entomol 9:223-226.

Bartelt RJ, Jones RL, Kulman HM. 1982. Hydrocarbon components of the yellowheaded spruce sawfly sex pheromone: A series of $(Z, Z)-9,19$ dienes. J Chem Ecol 8:95-114. 
Betke P, Ribbeck, R, Schmäschke R. 1991. Biological control of house flies with the antagonist Ophyra aenescens in animal production units. Proceedings of the 7th International Congress on Animal Hygiene. Leipzig (in German), p 504-515.

Brenner RJ, Carlson DA, Roth LM, Patterson RS. 1993. Morphological and chemotaxonomic identification of Blattella cockroaches (Blattaria: Blattellidae) from Taiwan and selected South Pacific basin locations. Invert Taxon 7:1205-1219.

Carlson DA. 1988a. Hydrocarbons for identification and phenetic comparisons: cockroaches, honey bees and tsetse flies. Florida Entomol 71:333-345.

Carlson DA. 1988b. Africanized and European honey-bee drones and comb waxes: Analysis of hydrocarbon components for identification. In: Needham GR, Page RF Jr, Delfinado-Baker M, Bowman CE, editors. Africanized honey bees and bee mites. New York: John Wiley and Sons, p 264-274.

Carlson DA, Brenner RJ. 1988. Cuticular hydrocarbons of North American Blattella for identification of all life stages. Ann Entomol Soc Am 81:711-723.

Carlson DA, Service MW. 1980. Identification of mosquitoes of Anopheles gambiae complex A \& B by analysis of cuticular components. Science 207:1089-1091.

Carlson DA, Nelson DR, Langley PA, Coates TW, Davis TL, Leegwater-Vander Linden M. 1984. Sex pheromone in Glossina pallidipes Austen: Identification and synthesis. J Chem Ecol 10:429-450.

Carlson DA, Roan C-S, Yost RA, Hector J. 1989. Dimethyl disulfide derivatives of long chain alkenes, alkadienes, and alkatrienes for gas chromatography/mass spectrometry. Anal Chem 61:1564-1571.

Carlson DA, Milstrey SK, Narang SK. 1993. Genetic classification of the tsetse flies using cuticular hydrocarbons. Bull Entomol Soc 83:507-515.

Carlson DA, Bernier UR, Sutton BD. 1998. Elution patterns of methyl-branched alkanes by capillary GC. J Chem Ecol 24:1845-1865.

Francis GW, Veland K. 1981. Alkylthiolation for the determination of double bond location in linear alkenes. $\mathrm{J}$ Chromatogr 219:379-384.

Hogsette JA, Jacobs RD. 1999. Failure of Hydrotaea aenescens, a larval predator of the house fly, Musca domestica, to establish in wet poultry manure on a commercial farm in Florida, U.S.A. MedVet Entomol 13:349-354.

Hogsette JA, Washington F. 1995. Quantitative mass production of Hydrotaea aenescens (Wiedemann) (Diptera: Muscidae). J Econ Entomol 88:1238-1242.

Lavine B, Carlson DA. 1987. European bee or Africanized bee? Species identification through chemical analysis. Anal Chem 59:469A-470A.
Lavine B, Carlson DA, Henry D, Jurs PD. 1988. Taxonomy based on chemical constituents: Differentiation of Africanized honey-bees from European honey-bees. J Chemometrics 2:29-37.

Nelson DR, Carlson DA. 1986. Cuticular hydrocarbons of the tsetse flies Glossina morsitans, G.austeni and $G$. pallidipes. Ins Biochem 16: 403-416.

Nelson DR, Sukkestad DR. 1970. Normal and branched aliphatic hydrocarbons from eggs of the tobacco hornworm. Biochemistry 9:4601-4611.

Nelson DR, Dillwith JW, Blomquist GJ. 1981. Cuticular hydrocarbons of the house fly, Musca domestica. Insect Biochem 11:187-197.

Nelson DR, Nunn NJ, Jackson L. 1984. Re-analysis of the methylalkanes of the grasshoppers, Melanoplus differentialis, M. packardii and $M$. sanguinipes. Insect Biochem 6:677-683.

Nelson DR, Carlson DA, Fatland CL. 1988. Cuticular hydrocarbons of the tsetse flies, II: G.p. palpalis, G.p. gambiensis, G. fuscipes, G. tachinoides and G. brevipalpis. J Chem Ecol 14:963-987.

Nelson DR, Fatland CL, Howard RW, McDaniel CA, Blomquist GJ. 1976. Re-analysis of the cuticular methylalkanes of Solenopsis invicta and S. richteri. Insect Biochem 10:163-168.

Nolan MP III, Kissam JB. 1985. Ophyra aenescens: A potential bio-control alternative for house fly control in poultry houses. J Agric Entomol 2:192-195.

Nolan MP III, Kissam JB. 1987. Nuisance potential of a dump fly, Ophyra aenescens (Diptera: Muscidae), breeding at poultry farms. Environ Entomol 16:828-831.

Ribbeck P, Betke P, Müller P, Schumann H, Heipe Th. 1987. Detrimental effects and control in intensive animal production. Monatsh Vet-Med 42:517-521 (in German).

Sutton BD, Carlson DA. 1993. Interspecific variation in Tephritid surface hydrocarbons. Arch Insect Biochem Physiol 23:53-65.

Sutton BD, Carlson DA. 1997a. Cuticular hydrocarbons of Glossina III: Subgenera Glossina and Nemorhina. J Chem Ecol 23:1291-1320.

Sutton BD, Carlson DA. 1997b. Cuticular hydrocarbon variation in the Tabanidae (Diptera): Tabanidus nigrovittatus complex of the North American Atlantic Coast. Ann Entomol Soc Am Systematics 90:542-549.

Turner EC Jr, Carter L. 1990. Mass rearing and introduction of Ophyra aenescens (Wiedemann) (Diptera: Muscidae) in high-rise caged layer houses to reduce house fly populations. J Agric Entomol 7:247-257.

Turner EC Jr, Ruszler PL, Dillon P, Carter L, Youngman R. 1992. An integrated pest management program to control house flies in commercial high rise houses. J Appl Poultry Res 1:242-250. 